\title{
Quality control of long-term mass-reared Aedes albopictus for population suppression
}

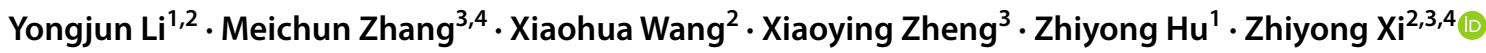

Received: 23 May 2020 / Revised: 16 January 2021 / Accepted: 19 January 2021 / Published online: 30 January 2021

(c) The Author(s) 2021

\begin{abstract}
The endosymbiotic bacterium Wolbachia is being developed as a tool to suppress mosquito populations and their transmitted pathogens, with successful field trials in multiple countries having resulted in efforts to scale up the capacity to mass-produce mosquitoes for release. However, major challenges exist to achieving this goal, including concerns that mass-reared mosquitoes will adapt to laboratory conditions during long-term maintenance and experience inbreeding depression, resulting in poor performance of the released mosquitoes in the field. Here, we assessed the performance of the Aedes albopictus $\mathrm{HC}$ line infected with a triple-strain Wolbachia after mass-rearing at scaled-up densities of up to 15 million mosquitoes per week for over 50 generations. In comparison with the wild-type GUA line, the HC mosquitoes had desirable characteristics for mass-rearing and release, including robust male mating competitiveness, high female reproductive capacity, reduced vector competence for dengue virus, and increased Wolbachia density. Although the larval survival rate of the HC and GUA lines was similar, the HC larvae developed significantly faster, possibly because of up-regulation of the molting hormone 20-hydroxyecdysone-related gene $E 75$ in the HC larvae. Our results indicate that over many generations mass-reared mosquito lines can retain their quality if large effective population sizes with sufficient genetic heterogeneity are maintained under optimized rearing conditions and demonstrate the long-term feasibility of deploying Wolbachia-based approaches for area-wide management of mosquito vectors for disease control.
\end{abstract}

Keywords Wolbachia $\cdot$ Mosquito $\cdot$ Mass-rearing $\cdot$ Dengue $\cdot$ Vector control

\section{Key message}

- Successful field trials have been accomplished to develop Wolbachia for mosquito suppression.

Communicated by Antonio Biondi.

Zhiyong Xi

xizy@msu.edu

1 Lingnan Statistical Science Research Institute, Guangzhou University, Guangzhou 510006, Guangdong, China

2 Guangzhou Wolbaki Biotech Co. Ltd., Guangzhou 510530, Guangdong, China

3 Sun Yat-Sen University - Michigan State University Joint Center of Vector Control for Tropical Diseases, Sun Yat-Sen University, Guangzhou 510080, Guangdong, China

4 Department of Microbiology and Molecular Genetics, Michigan State University, East Lansing, MI 48824, USA
- Aedes albopictus HC line carrying a novel Wolbachia has been mass-reared for $>50$ generations.

- Long-term mass-reared HC males maintain strong mating competitiveness and flight ability.

- Long-term mass-reared HC females maintain resistance to dengue virus, with life-span reduced.

- Long-term mass-reared HC larvae develop faster than their wild-type counterparts.

\section{Introduction}

Pathogens transmitted by mosquitoes, including malaria parasites, and dengue and Zika viruses, cause severe mortality and morbidity globally, but traditional vector control approaches are insufficient to protect humans from infection. Successful implementation of the sterile insect technique (SIT) for the control of several insect pests of agricultural and veterinary importance (Dyck et al. 2005) has encouraged significant efforts to develop analogous techniques for 
mosquito control (Harris et al. 2011; Mains et al. 2016). One of them is the incompatible insect technique (IIT), in which males infected with the maternally inherited endosymbiotic bacteria Wolbachia are released to induce incompatible matings with target females who are either uninfected or infected with different Wolbachia strains (Xi and Joshi 2016). IIT field trials have resulted in both historical success in eradicating Culex quinquefasciatus (Laven 1967) and recent success in effectively suppressing Aedes aegypti and Aedes albopictus (Baton et al. 2021; Crawford et al. 2020; Zheng et al. 2019).

Area-wide application of either IIT or SIT for mosquito vector control requires the capacity to scale up the production of incompatible/sterile males with robust mating competitiveness relative to the wild-type males. Despite significant efforts, mosquito mass-production has not yet attained the industrial scale reached by the operational SIT programs for screwworm and medfly control, two successful examples of the application of this technology (Dyck et al. 2005; Enkerlin et al. 2017). In contrast to the breeding and release of insects for screwworm and medfly control programs, there is a potential risk of arbovirus transmission among those involved in —or associated with—-the mass-rearing or massrelease of mosquitoes, either through escape of adult females within the rearing facility itself, or immediately following male release, if any contaminant females remain as a consequence of imperfect sex separation. It is neither ethical nor responsible to mass-produce and release mosquitoes with vector competence- the ability of a mosquito to acquire and ultimately transmit a pathogen-higher than wild-type mosquitoes. IIT has provided a solution to minimize this risk due to the ability of some Wolbachia strains to confer mosquito resistance to a broad spectrum of human pathogens (Bian et al. 2013, 2010; Kambris et al. 2009; Moreira et al. 2009).

We have recently been able to mass-rear more than 15 million mosquitoes per week enabling the weekly production and mass-release of $\sim 5$ million males of the transinfected Ae. albopictus $\mathrm{HC}$ line which has an artificial triple-strain Wolbachia infection (Zheng et al. 2019). As an essential component of any vector control program, quality control of the mass-reared $\mathrm{HC}$ mosquitoes has been extensively performed to ensure the robust fitness of the released mosquitoes relative to wild-type mosquitoes at the beginning of mass-rearing for the field trial (Zhang et al. 2015, 2016; Zheng et al. 2019). However, more than 50 generations have passed during the 4-year period since the $\mathrm{HC}$ line was first developed for mass-rearing, raising concerns that this mosquito line could have adapted to the artificial laboratory conditions, and/or have experienced rapid genetic changes and inbreeding depression, and, therefore, reduced performance in the field. Previous studies have found that laboratory-adapted Aedes mosquitoes show faster development and changes in body size (Ross et al. 2019; Yeap et al. 2013), increased sensitive to stress (Hoffmann and Ross 2018; Jong et al. 2017), and reduced male fecundity relative to their field counterparts (Ponlawat and Harrington 2007; Richardson and Williams 2013). Under laboratory settings, inbreeding was previously shown to be extremely costly to Ae. aegypti, resulting in reduced survivorship, increased development time, and lowered energy reserves for flight, dispersal, and basic metabolic functions (Koenraadt et al. 2010; Ross et al. 2019). Inbreeding depression could occur through mating between closely related individuals, resulting in an increase in homozygosity and the expression of deleterious traits (Reed and Frankham 2003; Wright et al. 2008). Loss of fitness in Ae. aegypti due to inbreeding correlated strongly with decreased effective population size, but, if population sizes were sufficiently high, colonized mosquitoes were able to maintain their fitness (Ross et al. 2019). Furthermore, interactions between Wolbachia and mosquito hosts might evolve under mass-rearing, resulting in reduction in both Wolbachia density and its mediated viral blocking effect, as evidenced by the previous observations that Wolbachia density was subject to environmental conditions of the mosquito larvae (Ross et al. 2017; Wiwatanaratanabutr and Kittayapong 2009). Thus, the early detection of reductions in fitness and other quality parameters of mass-reared mosquitoes resulting from laboratory adaptation and/or inbreeding depression are essential for the success of vector control programs involving mass-release of artificially bred insects.

In the present study, we evaluated key biological traits of HC mosquitoes, and compared them to the wild-type GUA strain, in order to examine the possibility that the HC mosquitoes are experiencing fitness loss as a result of longterm mass-rearing. Except for a minor reduction in female longevity, we found that $\mathrm{HC}$ mosquitoes otherwise retain key biological traits upon prolonged mass-rearing, including male mating competitiveness, Wolbachia-mediated virus blocking, and other life-history traits. These findings support the long-term feasibility of deploying IIT for area-wide management of mosquito vectors for disease control.

\section{Materials and methods}

\section{Mosquito colonies}

The Ae. albopictus $\mathrm{HC}$ line, infected with Wolbachia strains $w \mathrm{AlbA}, w \mathrm{AbB}$, and $w \mathrm{Pip}$, was produced at the mass-rearing factory run by Guangzhou Wolbaki Biotech Co., Ltd. (Wolbaki), according to a standard mass-rearing protocol (Zhang et al. 2017, 2018; Zheng et al. 2019), and is referred to as the "mHC" (i.e., mass-reared HC) line in this study. $\mathrm{mHC}$ had been mass-reared for over 50 generations at the time of the experiments reported here. The wild-type 
mosquito line GUA was recently established by collecting larvae from eight different field sites in Guangzhou (Supplementary Table 1). Approximately 200 larvae were collected from each location, then pooled together, and reared to adults $\left(G_{0}\right)$ under standard low-density laboratory conditions to establish the colony, which was used for experiments within five generations. Both mHC and GUA were reared under standard conditions during the experiment for measurement of biological traits. Larvae and adults were maintained in a standard mosquito rearing room at $27 \pm 2{ }^{\circ} \mathrm{C}$ and $75 \pm 5 \%$ relative humidity with a $12: 12$-h light:dark photoperiod. Adults were provided with fresh $10 \%$ sucrose solution, blood-fed on mice, and allowed to lay eggs, according to a protocol approved by the Ethics Committee on Laboratory Animal Care of the Zhongshan School of Medicine (approval number 2017-041).

\section{Male mating competitiveness}

Three replicate cages $(30 \mathrm{~cm} \times 30 \mathrm{~cm} \times 30 \mathrm{~cm})$ were each filled with 20 GUA and $20 \mathrm{mHC}$ virgin adult males 1-2 days post-eclosion. After $24 \mathrm{~h}, 20$ adult virgin GUA females of the same age as the males were also introduced into each replicate cage. Cages for an additional two groups,

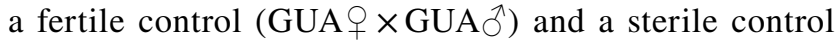
$\left(\mathrm{GUA}+\times \mathrm{mHC}^{\wedge}\right)$, were set up in parallel. Mosquitoes were kept in the cages for 3 days and then blood-fed on mice once. Three days later, oviposition cups were provided to the females for 2 days. After the eggs were collected and allowed to mature for an additional 5 days, they were hatched to determine the egg hatch rate, which was used to calculate Fried's Competitiveness Index (Fried 1971). Briefly, after the mean egg hatch rates of fertile control cages $\left(H_{\mathrm{n}}\right)$, sterile control cages $\left(H_{\mathrm{s}}\right)$, and treatment cages $\left(H_{\mathrm{o}}\right)$ were measured, the male mating competitiveness index (CI) was calculated using the equation: $\mathrm{CI}=\left[\left(H_{\mathrm{n}}-H_{\mathrm{o}}\right) /\left(H_{\mathrm{o}}-H_{\mathrm{s}}\right)\right] *(N / S)$, where $N$ and $S$ were the numbers of GUA and mHC males.

\section{Flight ability test}

Mosquito flight ability was measured using a modified flight test device (kindly provided by Jérémy Bouyer) (Fig. S1), according to a previously reported approach (Culbert et al. 2018) with minor modifications. In brief, GUA and $\mathrm{mHC}$ mosquitoes were reared to the pupal stage, and male pupae were collected and reared to the adult stage, with $10 \%$ sucrose solution provided once the adults had emerged. Approximately 100 males (2-3 days old) were collected using a mouth aspirator and transferred to the flight test tube. The fan above the tube was then turned on and allowed to run for $2 \mathrm{~h}$. The device was then placed in a $-20{ }^{\circ} \mathrm{C}$ freezer to kill all the tested mosquitoes. The number of mosquitoes outside the flight tube (the escaped group) and the number inside the tube (the non-escaped group) were recorded, and "the escape rate" was calculated as the number of escaped mosquitoes divided by the total number of tested mosquitoes (i.e., the proportion of all mosquitoes that escaped).

\section{Mosquito vector competence for dengue virus}

Mosquitoes were infected with dengue virus serotype 2 (DENV-2, New Guinea C strain) through blood-feeding as previously described (Bian et al. 2010). Fresh supernatant harvested from DENV-2-infected C6/36 cells was mixed 1:1 with human blood (final virus titer, $5 \times 10^{5} \mathrm{PFU} / \mathrm{ml}$ ), and then, the mixture was injected into feeders (Wolbaki) covered with pig intestine as a membrane. Feeders were connected to a water bath circulating system (Fisher) to keep the blood at $37^{\circ} \mathrm{C}$. Mosquitoes were allowed to feed on the mixture for 30-45 min. Only engorged mosquitoes were collected and maintained under standard rearing conditions. At 14 days post-blood-feeding, saliva samples were individually collected from twenty mosquitoes using the forced salivation technique, with minor modifications (Anderson et al. 2010): Mosquitoes were anesthetized with $\mathrm{CO}_{2}$, and their legs and wings were removed, and then the mosquito proboscis was inserted into a 10- $\mu$ l pipette tip containing $6 \mu \mathrm{l}$ of FBS and kept for $30 \mathrm{~min}$ at room temperature. The number of copies of DENV-2 in the saliva was quantified by RT-qPCR to reflect DENV-2 transmission potential of the mosquitoes.

\section{DNA extraction and quantification of Wolbachia density}

In order to minimize the influence of larval crowding on Wolbachia density, approximately 300 first-instar larvae were reared in a plastic tray $(36 \mathrm{~cm} \times 25 \mathrm{~cm} \times 5 \mathrm{~cm})$ with $1.5 \mathrm{~L} \mathrm{dH}_{2} \mathrm{O}$. Genomic DNA was extracted from 3 to 4 adult females ( 5 to 6 days post-eclosion) pooled together and homogenized in $500 \mu \mathrm{l}$ STE buffer with $20 \mu \mathrm{l}$ proteinase $\mathrm{K}(20 \mathrm{mg} / \mathrm{mL})$ and $70 \mu \mathrm{l}$ SDS (10\%) and then incubated at $55^{\circ} \mathrm{C}$ for $1 \mathrm{~h}$. DNA was then purified using phenol/chloroform extraction. $w$ AlbA-, $w$ AlbB-, and $w$ Pipspecific primers were used in real-time PCR to individually quantify each Wolbachia strain, as normalized by the reference gene ribosomal protein S6 (rps6). The total Wolbachia density was calculated as the sum of the individual densities of each of the three Wolbachia strains. Real-time PCR was performed using ChamQ SYBR qPCR Master Mix (Vazyme) on a Roche 96 machine with the following conditions: $95{ }^{\circ} \mathrm{C}$ for $30 \mathrm{~s}$, then 40 cycles of $90{ }^{\circ} \mathrm{C}$ for $5 \mathrm{~s}$, and $60^{\circ} \mathrm{C}$ for $30 \mathrm{~s}$, followed by melting curve analysis. The specific PCR products generated by each set of primers were cloned into the pMD-18T vector (Takara) to generate standard curves. Primers for rps6 (Zheng et al. 
2019) and $w s p$ of $w$ Pip (Liang et al. 2020), $w$ AlbA (Tortosa et al. 2008), and $w$ AlbB (Zheng et al. 2019) have been previously reported.

\section{Larval development and determination of sex ratio}

Eggs were immersed for $\sim 2 \mathrm{~h}$ into hatching solution: $1 \%$ $(\mathrm{w} / \mathrm{v})$ yeast powder in $\mathrm{dH}_{2} \mathrm{O}$. The resulting newly hatched first instar larvae were transferred to the larval rearing trays $(17 \mathrm{~cm} \times 11.8 \mathrm{~cm} \times 5.5 \mathrm{~cm})$ at a density of 100 larvae/300 mL water/tray. Liver powder was weighed and suspended in $5-10 \mathrm{ml}$ water and then provided to the $1 \mathrm{st}$ and $2 \mathrm{nd}$ instar larvae at $0.5 \mathrm{mg} / \mathrm{larvae} /$ day and $1.0 \mathrm{mg} /$ larvae/day for the 3rd and 4th instar larvae. From day 5, pupae were collected daily and sex-sorted until all larvae developed into pupae. Male and female pupae were distinguished by microscope examination of their terminalia (Benedict 2014), with the number of each sex recorded to measure the sex ratio. The larval survival rate was calculated as the number of pupae that developed divided by the total number of $1^{\text {st }}$ instar larvae and then multiplied by 100 so that it was expressed as a percentage.

\section{Assays for fecundity, egg hatch rate, and wing size}

After GUA and mHC pupae enclosed to adults, males were allowed to copulate with females for 5-7 days. Then, female mosquitoes were blood-fed with mice, and 62 engorged mosquitoes for each line were individually reared in a 50-ml plastic tube supplied with sugar and wet filter paper for laying eggs. Six days later, the filter papers were removed from the tubes and kept wet for 3 more days to allow the eggs to mature. Fecundity was measured by counting the number of eggs from each mosquito. Thirty randomly selected egg papers were individually immersed in hatching solution for $24 \mathrm{~h}$, and then egg hatch rate per individual female was calculated as the number of decapped eggs divided by the total number of eggs.

Wing length was used to estimate adult body size according to the approach reported previously, with a slight modification (Styer et al. 2007). In brief, wings were dissected from 5- to 7-day-old females and males (15 individuals of each sex), laid on glass slides, and then photographed together with a scale plate using a Cannon EOS $800 \mathrm{D}$ camera. The length $(\mathrm{mm})$ from the humeral cross vein to the wing tip was measured with Adobe Photoshop software, version CS6.

\section{Measurement of the relative expression of the 20-hydroxyecdysone (20E) primary response gene $E 75$ in mosquito larvae}

One hour after eggs were immersed in hatching solution, 500 each of newly hatched $\mathrm{mHC}$ and GUA larvae were transferred to rearing trays $(36 \mathrm{~cm} \times 25 \mathrm{~cm} \times 5 \mathrm{~cm}$, filled with about $2 \mathrm{~L}$ of water), with food provided as described above. At 48, 72, 96, and $128 \mathrm{~h}$ after egg hatching, five samples, each containing 10 to 20 larvae, were collected to measure the relative expression of $E 75$ in the two lines using the $2^{-\Delta \Delta \mathrm{Ct}}$ method (Schmittgen and Livak 2008).

\section{RNA extraction, reverse transcription, and qRT-PCR}

Total RNA from either mosquito saliva or larvae was extracted using RNAiso (Takara) according to the manufacturer's protocol. Extracted RNA was dissolved in RNase-free water, treated with gDNA wiper (Vazyme) at $42{ }^{\circ} \mathrm{C}$ for $2 \mathrm{~min}$, and then immediately reverse-transcribed using the HiScript Q RT SuperMix for qPCR (Vazyme). The resulting cDNA was stored at $-20{ }^{\circ} \mathrm{C}$ until used. Realtime PCR was performed using ChamQ SYBR qPCR Master Mix (Vazyme) on a Roche LightCycler 96 machine with the following conditions: $95{ }^{\circ} \mathrm{C}$ for $30 \mathrm{~s}$, then 40 cycles of $90{ }^{\circ} \mathrm{C}$ for $5 \mathrm{~s}$ and $60{ }^{\circ} \mathrm{C}$ for $30 \mathrm{~s}$, followed by melting curve analysis. In order to calculate the number of DENV-2 virus copies, the DENV-2 NS5 gene was cloned into the pMD-18T vector (Takara) as described previously (Zhang et al. 2013). A serial dilution of the recombinant plasmid was used to generate a standard curve. DENV-2 NS5 primers were newly designed: forward 5'-AACCAT GGAAGCTGTACGCA-3', reverse 5'-AGCTTCATCTCA CCTTGGGC-3'. The mosquito E75 expression level was normalized with $r p s 7$. Primers for $r p s 7$ have been previously reported (Zhang et al. 2013), and those for E75 were newly designed: forward 5'-CGCCTCCACTGTACGATA CC-3', reverse 5'-CCGCTCCCATACTGCTACTG-3'.

\section{Adult longevity assays}

Thirty newly emerged virgin male or female adults were transferred to small cages $(20 \mathrm{~cm} \times 20 \mathrm{~cm} \times 20 \mathrm{~cm})$ and kept under standard rearing conditions as described above. This setup was replicated three times for each sex of each mosquito line. Fresh $10 \%$ sucrose solution was provided to the mosquitoes. Every 2 days, the mosquitoes were checked, and dead mosquitoes were counted and removed until all mosquitoes had died. 


\section{Statistical analysis}

All data were statistically analyzed by SPSS 13.0 and GraphPad Prism 6.0 software. The normality of the datasets was checked using D'Agostino and Pearson omnibus normality test, and then, Student's $t$ test was used for data with normal distributions and Mann-Whitney $U$ test for data with nonnormal distributions. Larval development time was compared between mHC and GUA using a two-tailed Student's $t$ test. The relative expression level of $E 75$, male mating competitiveness, larval survival rate, sex ratio, adult size, fecundity, fertility, escape rate, and the number of DENV-2 NS5 copies in saliva samples were compared between $\mathrm{mHC}$ and GUA using two-tailed Mann-Whitney $U$ tests. The log-rank (Mantel-Cox) test was used to compare longevity within the two sexes between the two mosquito lines. DENV-2 prevalence in mosquito saliva samples was compared between mHC and GUA using Fisher's exact test.

\section{Results}

\section{Impact of long-term mass-rearing on the quality of adult HC males, in terms of mating competitiveness and flight ability}

In order to test whether long-term mass-rearing reduces the ability of $\mathrm{HC}$ males to mate with wild-type females, we measured the mating competitiveness of mass-reared HC males (mHC) using the GUA line recently established from the field. The results showed that the average mating competitiveness index of $\mathrm{mHC}$ males was $1.07 \pm 0.20$, and not significantly different from 1 , indicating that $\mathrm{mHC}$ males and GUA males showed equal mating competitiveness under laboratory conditions (Table 1).

We also compared the flight ability of mHC and GUA males by measuring, as an indirect indicator of male competitiveness, the capacity of mosquitoes to escape flight tubes (Culbert et al. 2018). There was no significant difference in the escape rates between $\mathrm{mHC}(0.86 \pm 0.03)$ and GUA males

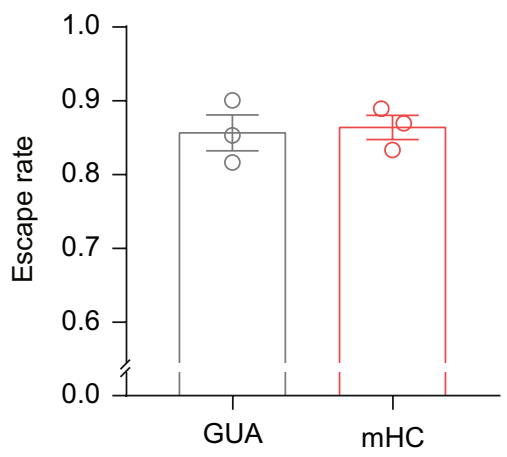

Fig. 1 Flight ability of $\mathrm{mHC}$ and GUA males. Flight ability was measured using a flight test device (as shown in Supplementary Fig. 1) and calculated as the "escape rate" (i.e., the proportion of all males that escaped their flight test tube). Each circle represents the value for a single replicate, and the bars shown are the means of the three replicates \pm SEM for each mosquito line. Mann-Whitney $U$ test, $P=0.827$

$(0.86 \pm 0.04)$ (Fig. 1), further supporting the inference that the mating competitiveness of $\mathrm{HC}$ males is not negatively affected by long-term mass-rearing.

\section{Impact of long-term mass-rearing on the quality of adult HC females, in terms of reproduction and vector competence}

Female reproductive quality, a key factor that determines the cost of mass production, can be reduced during long-term mass-rearing under factory conditions. Thus, we compared both the fecundity (i.e., the number of eggs produced by each female) and fertility (i.e., the proportion of eggs hatching) of $\mathrm{mHC}$ and GUA females. Again, we saw no significant difference in either fecundity ( $\mathrm{mHC} 77.95 \pm 3.6$ versus GUA $74.6 \pm 2.6$ eggs per female) or fertility ( $\mathrm{mHC} 0.84 \pm 0.02 \mathrm{vs}$ GUA $0.89 \pm 0.02$ hatch rate) between the two lines (Fig. 2a, b), indicating that there was no evidence of any impact of our mass-rearing conditions on the reproductive quality of $\mathrm{HC}$ females.

One advantage of using the $\mathrm{HC}$ line for IIT is that its artificial infection with the $w$ Pip Wolbachia strain increases
Table 1 Mating competitiveness index of mass-reared Ae. albopictus $\mathrm{HC}$ (mHC) males

\begin{tabular}{|c|c|c|c|c|}
\hline Mating groups & $\begin{array}{l}\text { Number of } \\
\text { replicates }\end{array}$ & Egg hatch rate $( \pm$ SEM $)$ & $\begin{array}{l}\text { Competitiveness } \\
\text { index (CI) }\end{array}$ & $P$ \\
\hline GUA $+\times\left(\right.$ GUA $\left.^{\lambda}: \mathrm{mHC}^{\lambda}\right)$ & 3 & $0.44 \pm 0.04\left(H_{\mathrm{o}}\right)$ & $1.07 \pm 0.20$ & 0.487 \\
\hline GUA $q \times \mathrm{GUA}^{\hat{}} \widehat{ }$ & 3 & $0.90 \pm 0.02\left(H_{\mathrm{n}}\right)$ & - & \\
\hline $\mathrm{GUA} q \times \mathrm{mHC} \widehat{0}$ & 3 & $0.00 \pm 0.00\left(H_{\mathrm{s}}\right)$ & - & \\
\hline
\end{tabular}

$H_{\mathrm{n}}$ mean egg hatch rate of fertile control cages; $H_{\mathrm{s}}$ mean egg hatch rate of sterile control cages; $H_{\mathrm{o}}$ mean egg hatch rate of treatment cages; CI male mating competitiveness index, calculated as: $\mathrm{CI}=\left[\left(H_{\mathrm{n}}-H_{\mathrm{o}}\right) /\right.$ $\left.\left(H_{\mathrm{o}}-H_{\mathrm{s}}\right)\right]^{*}(N / S)$, where $N$ and $S$ were the numbers of GUA and $\mathrm{mHC}$ males. The calculated CI value was compared with the theoretical value of 1 (i.e., no difference in mating competitiveness) using a MannWhitney $U$ test 


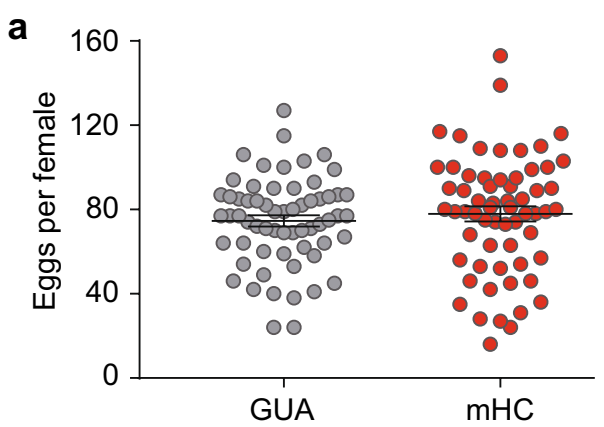

Fig. 2 Reproductive capacity of mHC and GUA females. a The number of eggs laid by each individual female $(n=62$ for each mosquito line). b The hatch rate of a subset of 30 individuals for each mos-

mosquito resistance to arbovirus infection (including that with both dengue and Zika viruses), so that any residual females resulting from imperfect sex separation, which are mixed with the males destined for field release, are less likely to be competent vectors (Zheng et al. 2019). The strength of mosquito resistance to arboviral infection is believed to be related to the density of Wolbachia in the somatic tissues of mosquitoes, which may be subject to changes during longterm mass-rearing. Therefore, we compared between $\mathrm{mHC}$ and GUA mosquitoes vector competence for dengue virus serotype 2 (DENV-2), which was previously used to assay the vector competence of the $\mathrm{HC}$ line when its mass-rearing

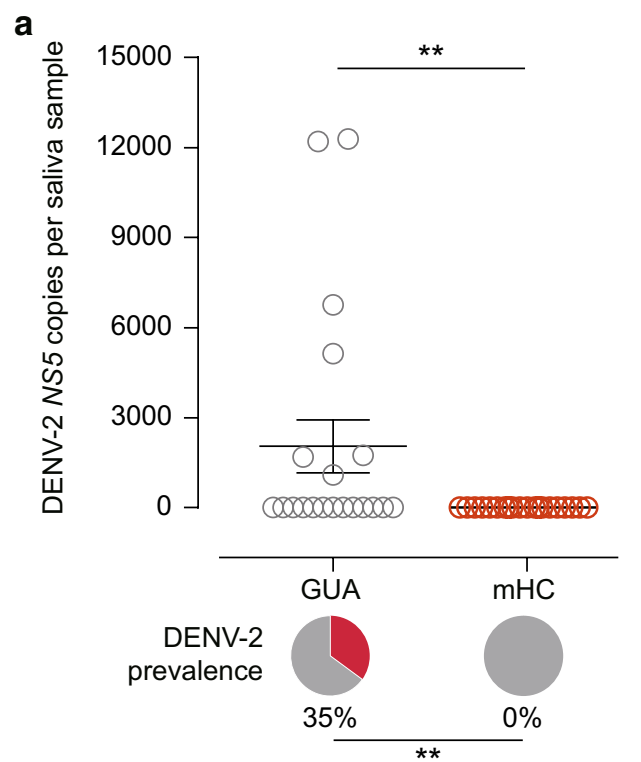

Fig. 3 Mosquito vector competence for dengue virus and Wolbachia density in mHC and GUA mosquitoes. a mHC and GUA mosquitoes were orally infected with DENV-2. At 14 days post-infection, individual saliva samples ( $n=20$ for each mosquito line) were collected, and DENV-2 genome copies were measured by RT-qPCR. B. Fiveto-six-day-old mHC and GUA females were collected $(n=8$ pools of b

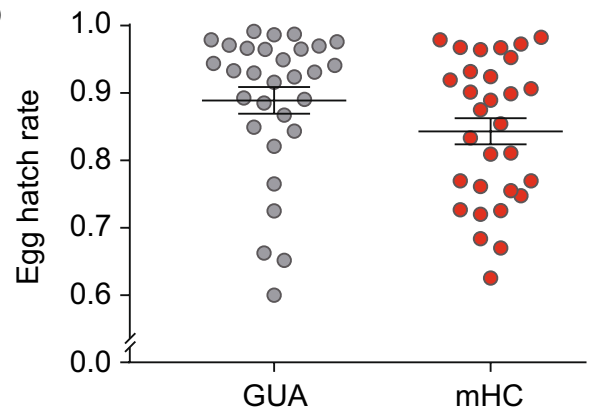

quito line randomly selected from those shown in a. Horizontal bars represent the mean \pm SEM. Mann-Whitney $U$ test: eggs per female, $P=0.31 ;$ egg hatch rate, $P=0.0682$

was first initiated (Zheng et al. 2019). Fourteen days after the mosquitoes were orally infected with DENV-2 virus, we collected their saliva and measured the copy numbers of the viral genomes. All of the mHC saliva samples were negative, whereas $35 \%$ of the GUA saliva samples were positive (Fig. 3a, Fisher's exact test, $P=0.008$ ). The density of Wolbachia in the whole bodies of 5-to-6-day-old adult females was also compared between the two mosquito $\mathrm{mHC}$ and GUA lines. The total Wolbachia density in the $\mathrm{mHC}$ females was about twofold higher than in the GUA females (Fig. 3b, Mann-Whitney $U$ test, $P=0.001$ ). While $w$ AlbB was dominant strain $(81 \%)$ in GUA, $w$ Pip counted for $88 \%$ of total

b

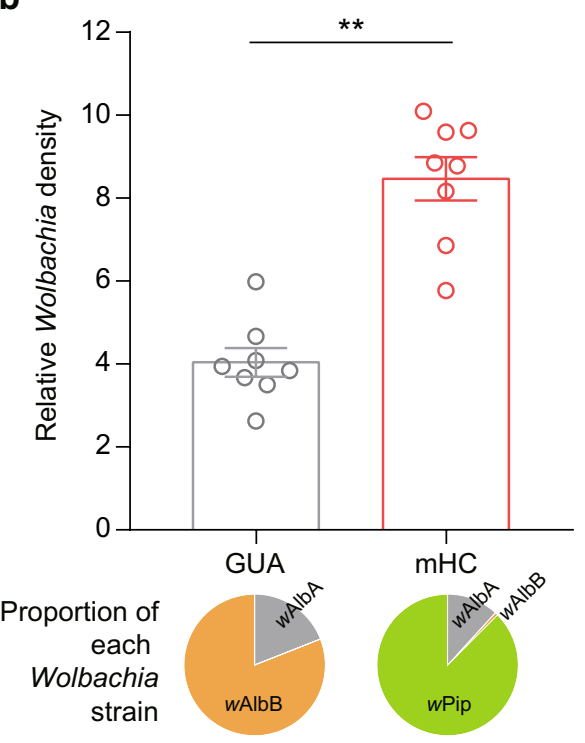

3 to 4 adults for each mosquito line), and the Wolbachia density was measured individually for each strain using qPCR. Mean \pm SEM and significance were determined using Mann-Whitney $U$ tests (for both DENV-2 copies and Wolbachia density) or Fisher's exact test (for the prevalence of DENV-2). $* * P<0.01$ 
Wolbachia density, with $w$ AlbB suppressed to a minimum in $\mathrm{mHC}$ (Fig. 3b). Overall, these results indicate that the Wolbachia-mediated virus-blocking effect, as well as high Wolbachia densities, was maintained during the long-term mass-rearing of the $\mathrm{HC}$ line.

\section{Impact of long-term mass-rearing on the quality of HC larvae, in terms of development and survivorship}

Long-term mass-rearing may impose selection on the development of mosquito larvae because the larvae are repeatedly reared under identical artificial conditions, with discrete generations produced according to a fixed schedule (e.g., pupae were always collected after the same amount of time had elapsed). We compared the larval development time and survivorship of the $\mathrm{mHC}$ and GUA lines and observed that the mHC larvae developed to pupae significantly more quickly than the GUA larvae. The average larval development time for both male $(5.13 \pm 0.04$ days $)$ and female $(5.73 \pm 0.02$ days) $\mathrm{mHC}$ was significantly shorter than the analogous times for GUA males $(5.54 \pm 0.08$ days) and females $(6.20 \pm 0.09$ days), respectively (Students' $t$ test, males: $t=7.93$, d.f. $=4, P=0.001$; females: $t=7.05$, d.f. $=4$, $P=0.002$, Fig. 4 a). However, there was no significant difference in the larval survival rate between the two lines (Mann-Whitney $U$ test, $P=0.7$, Fig. 4b).

Insect larval development is controlled by a critical steroid hormone, 20-hydroxyecdysone (20E), with the level of this hormone being positively correlated with the mRNA expression of the $20 \mathrm{E}$ primary response gene $E 75$ ( $\mathrm{Li}$ et al. 2019; Moeller et al. 2013). Therefore, we measured by qRTPCR the level of $E 75$ expression in larvae at $48,72,96$, and $120 \mathrm{~h}$ after egg hatching to determine whether E75 played a

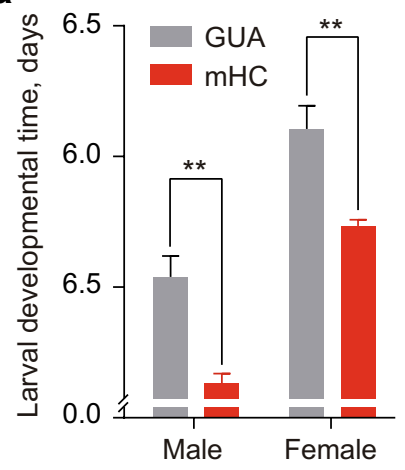

b

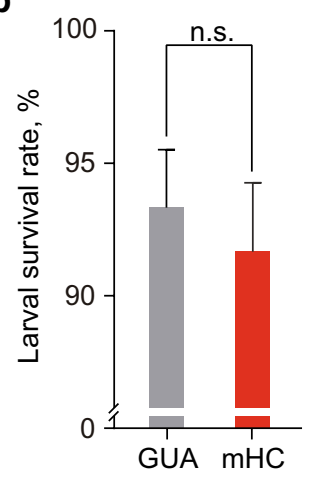

any role in promoting mHC larval development when compared to that of GUA larvae. We found that the expression of $E 75$ was significantly higher in mHC larvae than in GUA larvae at 96 or $120 \mathrm{~h}$ after egg hatching, but no significant difference was observed between the two lines at 48 or $76 \mathrm{~h}$ after egg hatching (Fig. 4c). These results suggest a potential correlation between rapid development to pupae and increased expression of $E 75$ during the latter developmental stages in mHC larvae.

\section{Impact of long-term mass-rearing on other HC adult life-history traits: sex ratio, body size, and longevity}

In order to further measure the impact of long-term massrearing on other mosquito life-history traits that might affect the efficacy of IIT-based suppression of target field populations, we also compared the sex ratio, body size, and longevity of mHC and GUA mosquitoes. We found no significant difference in either the sex ratio or adult wing (i.e., body) size between the mHC and GUA lines (Fig. 5a, b). However, mHC females (median longevity: 44 days) had significantly shorter life span than did GUA females (median longevity: 48 days) (Fig. $5 \mathrm{c}$, log-rank test, $P=0.0007$ ), but both mHC and GUA males had similar life spans (Fig. 5d, logrank test, $P>0.05$ ). Thus, long-term mass-rearing seems to exert a negative impact on $\mathrm{HC}$ longevity in a female-specific manner.

\section{Discussion}

The HC mosquito line passed rigorous quality control during the initial phase of its mass-rearing (Zhang et al. 2015, 2016; Zheng et al. 2019). In this work, we have here further

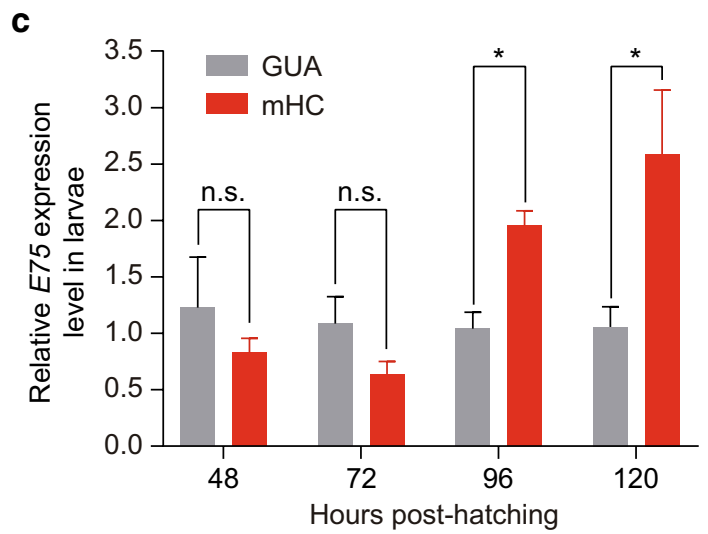

Fig. 4 Larval development and survival of $\mathrm{mHC}$ and GUA mosquitoes. a mHC larvae developed significantly faster than did GUA larvae $(n=3$ replicates). b Survival rate of $\mathrm{mHC}$ and GUA larvae $(n=3$ replicates). c E75 expression level in mosquito larvae at different times after egg hatching. Each time point shows data for five biological replicates of 10-20 larvae. Mean \pm SEM and significance were determined by using either a Student's $t$ test (A and B) or a MannWhitney $U$ test (C). n.s. not significant, ${ }^{*} P<0.05$; $* * P<0.01$ 


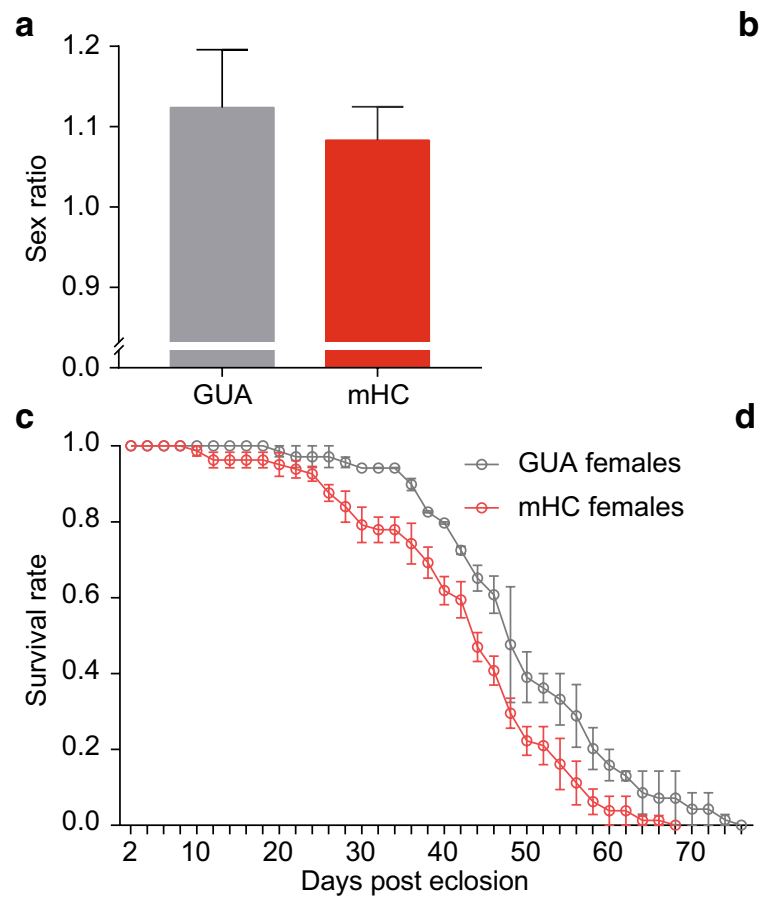

Fig. 5 Sex ratio, adult body size, and longevity of $\mathrm{mHC}$ and GUA mosquitoes. a Sex ratios; $n=3$ replicates. Mann-Whitney $U$ test, $P=0.7$. b Wing size.; $n=15$ individuals. Mann-Whitney $U$ test, males: $P=0.305$; females: $P=0.187$. c Female longev-

demonstrated that $\mathrm{HC}$ mosquitoes continue to show high performance, based on biological traits that are essential for the success of IIT-based population suppression, after massrearing in our factory facility for more than 50 generations. No differences were observed between mHC mosquitoes and their wild-type GUA counterparts in the following traits: the mating competitiveness and flight ability of males, fecundity and fertility of females, survivorship of larvae, and the sex ratio and body size of adults. As observed previously (Zheng et al. 2019), mHC females exhibit a lower vector competence for dengue virus than GUA. In addition, consistent with an elevated expression of $E 75$ at 96 and $120 \mathrm{~h}$ after egg hatching, the development of $\mathrm{HC}$ larvae to the pupal stage occurs significantly faster than GUA larvae. Despite the lack of any difference in the life span between HC and GUA adult males, adult GUA females live significantly longer than mHC females.

We have paid close attention to monitoring the quality of $\mathrm{HC}$ mosquitoes under mass-rearing conditions because previous studies have shown that long-term (from dozens of generations to more than 10 years) artificial maintenance in laboratory and/or factory settings can strongly reduce the fitness and/or heterozygosity of mosquitoes (i.e., inbreeding depression and/or adaptation to artificial environments) (Azrag et al. 2016; Jong et al. 2017; Koenraadt et al. 2010; Lainhart et al. 2015; Powell and Evans 2017; Ross et al. b

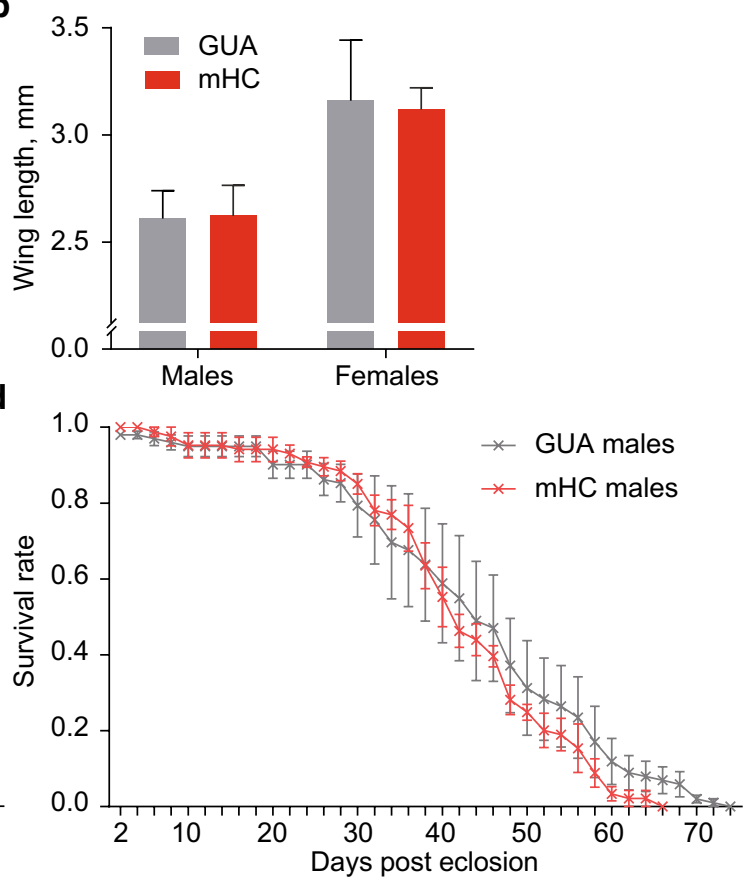

ity. Mean \pm SEM; $n=3$ replicates. Log-rank (Mantel-Cox) test, $P=0.0007$. d Male longevity; $n=3$ replicates. Log-rank (MantelCox) test, $P>0.05$. All figures show the mean \pm SEM

2019), with the potential to cause a reduction in the quality of artificially bred mosquitoes relative to wild-type mosquitoes in target field populations (Hoffmann and Ross 2018; Reed and Frankham 2003; Ross et al. 2019). These negative impacts have not been observed in $\mathrm{HC}$ mosquitoes reared under our mass-rearing protocol for more than 50 generations. This may be explained by the three reasons. First, before being used in mass-rearing, the $\mathrm{HC}$ mosquitoes had been repeatedly outcrossed for seven generations with the wild-type GUA line, established from the field by pooling collected samples from 20 different locations in Guangzhou (Zhang et al. 2015; Zheng et al. 2019). This outcrossing enabled us to introduce considerable genetic heterogeneity into the $\mathrm{HC}$ mosquitoes and improved their fitness, as observed in Ae. aegypti (Ross et al. 2019; Yeap et al. 2011). Second, after outcrossing, the $\mathrm{HC}$ mosquitoes were maintained at a large population size (millions of mosquitoes in each generation), thus greatly reducing the possibility of any inbreeding depression that might occur during mass-rearing (Ross et al. 2019). Third, each step of our mass-rearing protocol has been optimized-including the density of larvae in the rearing trays, the sex ratio and density of adults in each cage, and the egg storage conditions-in order to minimize the stress to the mosquitoes as much as possible (Zhang et al. 2017, 2018). Although it remains speculative at this stage, the Wolbachia themselves may provide certain benefits to HC 
mosquitoes under mass-rearing conditions. We have consistently observed that $\mathrm{HC}$ larvae develop faster than GUA larvae (Zhang et al. 2015), as supported by the increased expression of molting hormone regulating $E 75$ gene in the $\mathrm{HC}$ larvae. Overexpression of $E 75$ gene is positively correlated with $20 \mathrm{E}$ (ecdysteroid) and related to synthesis of chitin and metamorphosis ( $\mathrm{Li}$ et al. 2016; Liu et al. 2018). $E 75$ has its highest peak of activity during the last stages of larval development prior to pupae formation (Margam et al. 2006), which is consistent with observations of this study. It would be interesting to explore if the overexpression of $E 75$ is induced by the triple-strain Wolbachia infection of $\mathrm{mHC}$ ( $w \mathrm{AlbA}, w \mathrm{AbB}$, and $w \mathrm{Pip}$ ).

The lack of evident fitness reduction under laboratory conditions in our mass-reared $\mathrm{HC}$ mosquitoes suggests that, with rearing conditions optimized, long-term mass-rearing does not necessarily result in reduced quality of insects produced for mass-release in vector control programs. It was reported that a 40-year-old colonized line of the tsetse fly Glossina palpalis gambiensis was still competitive in a field trial in Burkina-Faso (Sow et al. 2012), and this line outcompeted a recently colonized strain from the target area in Senegal (Bassene et al. 2017), indicating that long-term, large-scale breeding of insects does not necessarily have adverse effects on male mating competitiveness.

It is also possible that the 50-generation (4-year) massrearing is not of sufficiently long duration for detrimental effects to accumulate to the extent that can be detected by our experimental approaches. If this is the case, continuous monitoring of the essential traits of mass-reared HC mosquitoes will be necessary for quality control. Theoretically, inbreeding-related colony depression can be reversed by outcrossing with wild-type mosquitoes (Ekechukwu et al. 2015). If inbreeding depression of the HC line does occur in the future, we can outcross this line with field-collected $A e$. albopictus males to quickly restore the level of fitness, since the triply Wolbachia-infected $\mathrm{HC}$ line causes unidirectional cytoplasmic incompatibility when these mosquitoes mate with wild-type mosquitoes (Zheng et al. 2019). This is one advantage of HC mosquitoes, as opposed to other transinfected lines that induce bi-directional cytoplasmic incompatibility with wild-type mosquitoes, and for whom removing native Wolbachia from field mosquitoes is required before outcrossing.

Our results show that the native Wolbachia strain wAlbB in the HC line is strongly suppressed to a very low level, suggesting the presence of competition between the two strains in the supergroup B ( $w$ Pip and $w \mathrm{AlbB}$ ), which infect HC. A similar result was also observed in another Ae. albopictus line carrying a triple-strain Wolbachia infection, in which $w \mathrm{Mel}$ inhibited $w \mathrm{AlbA}$, indicating competition between two strains in the supergroup A (Liang et al. 2020). It is worth noting that the $\mathrm{HC}$ line expresses unidirectional cytoplasmic incompatibility when it is crossed with wild-type mosquitoes (Zheng et al. 2019), indicating that even the low level of $w \mathrm{AlbB}$ in $\mathrm{HC}$ eggs is sufficient to rescue sperm modification.

As it was technically challenging to mass-rear GUA at the same scale as mHC, comparisons of these two lines were performed under the standard laboratory condition in this study. Thus, our results mainly reflect the intrinsic properties of the two mosquito lines, and different results may be observed if they are compared under mass-rearing conditions. It is also possible that the approach used in the current study only reflects the level of quality of traits such as male mating competitiveness in the setting of the laboratory, and these levels may not represent the real situation in the field (Soemori et al. 1980). Previous studies showed that a transgenic line of Ae. aegypti with female-specific flightless phenotype performed well in laboratory experiments but was not competitive when tested in semi-field experiments (Facchinelli et al. 2013), while mating competitiveness of another transgenic line observed in the field was much lower than results from laboratory and larger semi-field mating cage studies (Carvalho et al. 2015). Conversely, another study showed that the field-derived males had decreased mating vigor when they were confined in a small cage to which mass-reared males had been adapted, demonstrating that "control" wild-type insects might also exhibit lower quality in experimental laboratory settings (Soemori et al. 1980). Future studies should be performed to compare male mating competitiveness in semi-field cages and field settings.

Unlike a previous report of no difference in life span between HC and GUA females (Zhang et al. 2015), we saw a significant reduction in the longevity of $\mathrm{HC}$ females when compared to GUA females when both lines were fed on sugar alone. It is unknown whether this is caused by a reduction in genetic variability of $\mathrm{HC}$ mosquitoes during the long-term mass-rearing. Augmented fertility and fecundity accompanied by increased longevity was previously reported for Ae. albopictus infected with $w \mathrm{AlbA}$ and $w$ AlbB (Dobson et al. 2002). Further studies will investigate whether this difference is caused by a change in Wolbachia composition and abundance, and whether a different result will be obtained when a bloodmeal is provided to the females (Joshi et al. 2014). It is worth noting that reduced female longevity decreases the risk of viral transmission associated with unintentional release of residual females into the field.

Overall, our results highlight the robustness of the biological traits in $\mathrm{HC}$ mosquitoes under long-term mass-rearing conditions, which can affect both the mass-rearing efficacy and male mating performance in the field, thus encouraging further development and deployment of Wolbachia-based IIT for mosquito vector control. The effort to maintain sufficient genetic heterogeneity in a large population under optimized rearing conditions is intended to impose less stress on mosquito 
colony and may contribute to avoiding the problem of inbreeding depression and reductions in insect quality. It would be interesting to further understand Wolbachia-mosquito interactions in relation to the maintenance of strong mating competitiveness in $\mathrm{HC}$ males, the high level of viral blocking in $\mathrm{HC}$ females, and the rapid development of $\mathrm{HC}$ larvae. Our future work includes developing rapid, sensitive assays that can be used in both laboratory conditions and field settings for quality control and early diagnosis of fitness loss, as well as novel treatment approaches for boosting mosquito quality when colony depression occurs. Further efforts will also be made to explore novel Wolbachia strains and their combinations in order to develop the next generation of transinfected lines that are best suitable for both mass production and field conditions, in terms of cost-effectiveness in operation, robustness, and stability of desired characteristics in various environments.

\section{Author contributions}

$\mathrm{YL}$ and $\mathrm{ZX}$ conceived the idea and designed the experiments. $\mathrm{ZX}, \mathrm{ZH}$, and $\mathrm{XZ}$ supervised the project. YL performed the majority of the experiments and analyzed the data. MZ and $\mathrm{XW}$ performed the remaining experiments. YL and ZX wrote the manuscript.

Supplementary Information The online version of this article (https:// doi.org/10.1007/s10340-021-01340-z) contains supplementary material, which is available to authorized users. Acknowledgements We thank Drs. Jérémy Bouyer and Mamai Wadaka for their technical assistance, and Drs. Deborah McClellan and Luke Anthony Baton for editorial assistance.

Funding This work was supported by Scientific and Technological Leading Talents of Guangzhou Development District (No. 2013L-P116), Guangdong Innovative Research Team Program (No. 2011S009), and a Strategic Partnership Grant from Michigan State University.

Availability of data and materials All datasets presented in this study are included in the article/Supplementary Material.

\section{Compliance with ethical standards}

Conflict of interest Y. L., X.W., and Z.X. are affiliated with Guangzhou Wolbaki Biotech Co., Ltd.

Ethical approval Mosquitoes were fed on the blood of anesthetized mice according to a protocol approved by the Ethics Committee on Laboratory Animal Care of the Zhongshan School of Medicine (Approval Number 2017-041).

Informed consent Informed consent was obtained from all co-authors of the study.

Open Access This article is licensed under a Creative Commons Attribution 4.0 International License, which permits use, sharing, adaptation, distribution and reproduction in any medium or format, as long as you give appropriate credit to the original author(s) and the source, provide a link to the Creative Commons licence, and indicate if changes were made. The images or other third party material in this article are included in the article's Creative Commons licence, unless indicated otherwise in a credit line to the material. If material is not included in the article's Creative Commons licence and your intended use is not permitted by statutory regulation or exceeds the permitted use, you will need to obtain permission directly from the copyright holder. To view a copy of this licence, visit http://creativecommons .org/licenses/by/4.0/.

\section{References}

Anderson SL, Richards SL, Smartt CT (2010) A simple method for determining arbovirus transmission in mosquitoes. J Am Mosq Control Assoc 26:108-111. https://doi.org/10.2987/09-5935.1

Azrag RS, Ibrahim K, Malcolm C, Rayah EE, El-Sayed B (2016) Laboratory rearing of Anopheles arabiensis: impact on genetic variability and implications for Sterile Insect Technique (SIT) based mosquito control in northern Sudan. Malar J 15:432. https://doi. org/10.1186/s12936-016-1484-2

Bassene MD et al (2017) Competitiveness and survival of two strains of Glossina palpalis gambiensis in an urban area of Senegal. PLoS Negl Trop Dis 11:e0006172. https://doi.org/10.1371/journ al.pntd.0006172

Baton LA, Zhang D, Li Y, Xi Z (2021) Combining the incompatible and sterile insect techniques for pest and vector control. In: Hendrichs J, Vreysen Pereira R, MJB, (eds) Area-wide integrated pest management: development and field application. CRC Press, Boca Raton, FL (in press)

Benedict MQ (2014) Methods in Anopheles research, Chapter 3: specific Anopheles techniques

Bian G, Xu Y, Lu P, Xie Y, Xi Z (2010) The endosymbiotic bacterium Wolbachia induces resistance to dengue virus in Aedes aegypti. PLoS Pathog 6:e1000833

Bian G et al (2013) Wolbachia invades Anopheles stephensi populations and induces refractoriness to Plasmodium infection. Science 340:748-751. https://doi.org/10.1126/science.1236192

Carvalho DO et al (2015) Suppression of a field population of Aedes aegypti in Brazil by sustained release of transgenic male mosquitoes. PLoS Negl Trop Dis 9:e0003864. https://doi.org/10.1371/ journal.pntd.0003864

Crawford JE et al (2020) Efficient production of male Wolbachiainfected Aedes aegypti mosquitoes enables large-scale suppression of wild populations. Nat Biotechnol 38:482-492. https://doi. org/10.1038/s41587-020-0471-x

Culbert NJ, Balestrino F, Dor A, Herranz GS, Yamada H, Wallner T, Bouyer J (2018) A rapid quality control test to foster the development of genetic control in mosquitoes. Sci Rep 8:16179. https:// doi.org/10.1038/s41598-018-34469-6

Dobson SL, Marsland EJ, Rattanadechakul W (2002) Mutualistic Wolbachia infection in Aedes albopictus: accelerating cytoplasmic drive. Genetics 160:1087-1094

Dyck VA, Hendrichs J, Robinson AS (2005) Sterile insect technique: principles and practice in area-wide integrated pest management. Springer, Dordrecht

Ekechukwu NE, Baeshen R, Traore SF, Coulibaly M, Diabate A, Catteruccia F, Tripet F (2015) Heterosis increases fertility, fecundity, and survival of laboratory-produced $\mathrm{F} 1$ hybrid males of the malaria mosquito Anopheles coluzzii. G3 (Bethesda) 5:26932709. https://doi.org/10.1534/g3.115.021436

Enkerlin WR, Gutiérrez Ruelas JM, Pantaleon R, Soto Litera C, Villaseñor Cortés A, Zavala López JL (2017) The Moscamed 
Regional Programme: review of a success story of area-wide sterile insect technique application. Entomol Exp Appl 164:188-203. https://doi.org/10.1111/eea.12611

Facchinelli L et al (2013) Field cage studies and progressive evaluation of genetically-engineered mosquitoes. PLoS Negl Trop Dis 7:e2001. https://doi.org/10.1371/journal.pntd.0002001

Fried M (1971) Determination of sterile-insect competitiveness. J Econ Entomol 64:869-872

Harris AF et al (2011) Field performance of engineered male mosquitoes. Nat Biotechnol 29:1034-1037. https://doi.org/10.1038/ nbt.2019

Hoffmann AA, Ross PA (2018) Rates and patterns of laboratory adaptation in (mostly) insects. J Econ Entomol 111:501-509. https:// doi.org/10.1093/jee/toy024

Jong ZW, Kassim NFA, Naziri MA, Webb CE (2017) The effect of inbreeding and larval feeding regime on immature development of Aedes albopictus. J Vector Ecol 42:105-112. https://doi. org/10.1111/jvec. 12244

Joshi D, McFadden MJ, Bevins D, Zhang F, Xi Z (2014) Wolbachia strain $w$ AlbB confers both fitness costs and benefit on Anopheles stephensi. Parasit Vectors 7:336. https://doi. org/10.1186/1756-3305-7-336

Kambris Z, Cook PE, Phuc HK, Sinkins SP (2009) Immune activation by life-shortening Wolbachia and reduced filarial competence in mosquitoes. Science 326:134-136

Koenraadt CJ, Kormaksson M, Harrington LC (2010) Effects of inbreeding and genetic modification on Aedes aegypti larval competition and adult energy reserves. Parasit Vectors 3:92. https:// doi.org/10.1186/1756-3305-3-92

Lainhart W, Bickersmith SA, Moreno M, Rios CT, Vinetz JM, Conn JE (2015) Changes in genetic diversity from field to laboratory during colonization of Anopheles darlingi Root (Diptera: Culicidae). Am J Trop Med Hyg 93:998-1001. https://doi.org/10.4269/ ajtmh.15-0336

Laven H (1967) Eradication of Culex pipiens fatigans through cytoplasmic incompatibility. Nature 216:383-384

Li K et al (2016) 20-Hydroxyecdysone (20E) primary response gene E75 isoforms mediate steroidogenesis autoregulation and regulate developmental timing in bombyx. J Biol Chem 291:18163-18175. https://doi.org/10.1074/jbc.M116.737072

Li K, Jia QQ, Li S (2019) Juvenile hormone signalling — a mini review. Insect Sci 26:600-606. https://doi.org/10.1111/1744-7917.12614

Liang X, Liu J, Bian G, Xi Z (2020) Wolbachia inter-strain competition and inhibition of expression of cytoplasmic incompatibility in mosquito. Front Microbiol 11:1638. https://doi.org/10.3389/ fmicb.2020.01638

Liu XJ, Sun YW, Li DQ, Li S, Ma EB, Zhang JZ (2018) Identification of LmUAP1 as a 20-hydroxyecdysone response gene in the chitin biosynthesis pathway from the migratory locust, Locusta migratoria. Insect Sci 25:211-221. https://doi. org/10.1111/1744-7917.12406

Mains JW, Brelsfoard CL, Rose RI, Dobson SL (2016) Female adult Aedes albopictus suppression by Wolbachia-infected male mosquitoes. Sci Rep 6:33846. https://doi.org/10.1038/srep33846

Margam VM, Gelman DB, Palli SR (2006) Ecdysteroid titers and developmental expression of ecdysteroid-regulated genes during metamorphosis of the yellow fever mosquito, Aedes aegypti (Diptera: Culicidae). J Insect Physiol 52:558-568. https://doi. org/10.1016/j.jinsphys.2006.02.003

Moeller ME, Danielsen ET, Herder R, O'Connor MB, Rewitz KF (2013) Dynamic feedback circuits function as a switch for shaping a maturation-inducing steroid pulse in Drosophila. Development 140:4730-4739. https://doi.org/10.1242/dev.099739

Moreira LA et al (2009) A Wolbachia symbiont in Aedes aegypti limits infection with dengue, Chikungunya, and Plasmodium. Cell 139:1268-1278
Ponlawat A, Harrington LC (2007) Age and body size influence male sperm capacity of the dengue vector Aedes aegypti (Diptera: Culicidae). J Med Entomol 44:422-426. https://doi.org/10.1603/00222585(2007)44[422:aabsim]2.0.co;2

Powell JR, Evans BR (2017) How much does inbreeding reduce heterozygosity? Empirical results from Aedes aegypti. Am J Trop Med Hyg 96:157-158. https://doi.org/10.4269/ajtmh.16-0693

Reed DH, Frankham R (2003) Correlation between fitness and genetic diversity. Conserv Biol 17:230-237. https://doi.org/10.104 6/j.1523-1739.2003.01236.x

Richardson AJ, Williams CR (2013) Inter-population mating success in Australian dengue vector mosquitoes: effects of laboratory colonization and implications for the spread of transgenics. J Vector Ecol 38:111-119. https://doi.org/10.1111/j.1948-7134.2013.12016.x

Ross PA, Wiwatanaratanabutr I, Axford JK, White VL, EndersbyHarshman NM, Hoffmann AA (2017) Wolbachia infections in Aedes aegypti differ markedly in their response to cyclical heat stress. PLoS Pathog 13:e1006006. https://doi.org/10.1371/journ al.ppat.1006006

Ross PA, Endersby-Harshman NM, Hoffmann AA (2019) A comprehensive assessment of inbreeding and laboratory adaptation in Aedes aegypti mosquitoes. Evol Appl 12:572-586. https://doi. org/10.1111/eva.12740

Schmittgen TD, Livak KJ (2008) Analyzing real-time PCR data by the comparative $\mathrm{C}(\mathrm{T})$ method. Nat Protoc 3:1101-1108. https://doi. org/10.1038/nprot.2008.73

Soemori H, Tsukaguchi S, Nakamori H (1980) Comparison of mating ability and mating competitiveness between mass-reared and wild strains of the melon fly, Dacus cucurbitae Coquillett (Diptera: Tephritidae). Jpn J Appl Entomol Zool 24:246-250

Sow A et al (2012) Irradiated male tsetse from a 40-year-old colony are still competitive in a Riparian forest in Burkina Faso. PLoS One 7:e37124. https://doi.org/10.1371/journal.pone.0037124

Styer LM, Meola MA, Kramer LD (2007) West Nile virus infection decreases fecundity of Culex tarsalis females. J Med Entomol 44:1074-1085

Tortosa P, Courtiol A, Moutailler S, Failloux A, Weill M (2008) Chikungunya-Wolbachia interplay in Aedes albopictus. Insect Mol Biol 17:677-684

Wiwatanaratanabutr I, Kittayapong P (2009) Effects of crowding and temperature on Wolbachia infection density among life cycle stages of Aedes albopictus. J Invertebr Pathol 102:220-224. https ://doi.org/10.1016/j.jip.2009.08.009

Wright LI, Tregenza T, Hosken DJ (2008) Inbreeding, inbreeding depression and extinction. Conserv Genet 9:833

Xi Z, Joshi D (2016) Genetic control of malaria and dengue using Wolbachia. In: Adelman ZN (ed) Genetic control of malaria and dengue. Elsevier Inc, Amsterdam, pp 305-333

Yeap HL et al (2011) Dynamics of the "popcorn" Wolbachia infection in outbred Aedes aegyptiinforms prospects for mosquito vector control. Genetics 187:583-595. https://doi.org/10.1534/genet ics. 110.122390

Yeap HL, Endersby NM, Johnson PH, Ritchie SA, Hoffmann AA (2013) Body size and wing shape measurements as quality indicators of Aedes aegypti mosquitoes destined for field release. Am J Trop Med Hyg 89:78-92. https://doi.org/10.4269/ajtmh.12-0719

Zhang M et al (2013) Differential proteomics of Aedes albopictus salivary gland, midgut and C6/36 cell induced by dengue virus infection. Virology 444:109-118. https://doi.org/10.1016/j.virol .2013.06.001

Zhang D, Zheng X, Xi Z, Bourtzis K, Gilles JR (2015) Combining the sterile insect technique with the incompatible insect technique: I-impact of Wolbachia infection on the fitness of triple- and double-infected strains of Aedes albopictus. PLoS ONE 10:e0121126. https://doi.org/10.1371/journal.pone.0121126 
Zhang D, Lees RS, Xi Z, Bourtzis K, Gilles JR (2016) Combining the sterile insect technique with the incompatible insect technique: III-robust mating competitiveness of irradiated triple Wolbachia-infected Aedes albopictus males under semi-field conditions. PLoS ONE 11:e0151864. https://doi.org/10.1371/ journal.pone.0151864

Zhang D et al (2017) Establishment of a medium-scale mosquito facility: optimization of the larval mass-rearing unit for Aedes albopictus (Diptera: Culicidae). Parasit Vectors 10:569. https:// doi.org/10.1186/s13071-017-2511-z

Zhang D et al (2018) Establishment of a medium-scale mosquito facility: tests on mass production cages for Aedes albopictus (Diptera:
Culicidae). Parasit Vectors 11:189. https://doi.org/10.1186/s1307 1-018-2750-7

Zheng $X$ et al (2019) Incompatible and sterile insect techniques combined eliminate mosquitoes. Nature 572:56-61. https://doi. org/10.1038/s41586-019-1407-9

Publisher's Note Springer Nature remains neutral with regard to jurisdictional claims in published maps and institutional affiliations. 